\title{
Is more always better? Simulating Feedback Exchange in Organizations
}

\author{
Sacha Fuchs ${ }^{1}$, Roman Rietsche ${ }^{1}$, Stephan Aier ${ }^{1}$, and Michael Rivera ${ }^{2}$ \\ ${ }^{1}$ University of St. Gallen, Institute of Information Management, St. Gallen, Switzerland \\ \{sacha.fuchs, roman.rietsche, stephan.aier\}@unisg.ch \\ ${ }^{2}$ Department of Strategic Management, Temple University, Philadelphia, Pennsylvania, USA \\ \{rivera\}@temple.edu
}

\begin{abstract}
More and more employees request feedback from their organizations to develop and learn. This is reflected by a growing number of digital feedback apps which facilitate high-frequency feedback exchange. However, the effect of feedback has hardly been studied on an organizational level due to complexity. Therefore, we strive to analyze organizational feedback exchange with an agentbased simulation model. Concretely, we study the effect of feedback length and feedback frequency on the organizational return on investment (ROI) of feedback exchange. Our study shows that feedback length stays in an inverted U-shape relationship with ROI. Contrarily, feedback frequency is negatively correlated with ROI. When analyzed jointly, two sweet spots arise: one for medium-length, frequent feedback, and the other, for longer infrequent feedback.
\end{abstract}

Keywords: Organizational feedback exchange, feedback app, return on investment, simulation, agent-based modeling

\section{Introduction}

Employees and the generation Y request more and more feedback from their managers [1]. Additionally, they demand instant responses which they are used to from social media platforms [1]. This call for new forms of feedback is clearly reflected by the increasing number of digital feedback apps that facilitate more frequent feedback exchange [2]. For example, workstream collaboration solutions like Slack, Skype, MS Teams or standalone feedback apps like DevelapMe ${ }^{1}$, Lattice $^{2}, 15$ Five $^{3}$, offer a wide array of mechanisms that can be used to facilitate feedback in organizations [3].

\footnotetext{
${ }^{1} \mathrm{https}: / /$ www.develapme.com/

${ }^{2} \mathrm{https}: / /$ lattice.com/

${ }^{3}$ https://www.15five.com/
} 
But why are organizations concerned with providing feedback to their employees? Building upon the insight that employees can be a key component of competitive advantage [4], the improvement of existing work practices is of high relevance [5]. One method for helping employees to improve their work practices, is constructive and timely feedback. The existing body of knowledge highlights the strategic value of feedback as an essential driver of employee motivation, learning and development [6, 7]. Feedback helps improve employees' performance, when they anticipate, seek, receive, process, react to, and finally use feedback to adjust their practices [8].

However, the effectiveness of feedback is dependent on its structure and content as it determines the receivers reaction [9]. A feedback message comprises the content [10], its timing [11] and the form of delivery [12]. The study at hand focuses on two of those components in the given context of digital feedback apps. First, the feedback content which is at the core of any feedback. Specific feedback helps employees improve, but if the message is too long, employees might ignore it [11]. Therefore, in this study we analyze the feedback length as a proxy for several content dimensions. Second, we explore the effect of feedback frequency, which is a highly discussed topic in literature and practice. In the past feedback was seen as an annual management process such as managers provide feedback to their employees once-a-year [13]. However, this approach has been criticized for a long time $[2,14,15]$ as in "the world isn't really on an annual cycle anymore for anything" [16].

The trend of more and more feedback has hardly been challenged in the literature, since measuring this effect on an organizational level is highly complex and problematic as components of the feedback process are interdependent and depend on organizational characteristics [9]. Hence, the question arises as how much feedback is necessary and beneficial for organizations. Previous studies were predominantly focused on an individual level of analysis to build a comprehensive understanding around the concept of feedback. These efforts have led to an extensive body of literature that explains the processes, components, and advantages of feedback. For example, feedback characteristics [9], behavior reactions to feedback $[6,17,18]$ and feedback efficiency [14]. 
In fact, the effects of feedback on an organizational level could only be studied within the constraints of empirical settings. However, the strong conceptual basis allows us to overcome those constraints and to explore the organizational effects of feedback through well-grounded computer simulation experiments. Specifically, agentbased modeling can be used to model emergent phenomena stemming from interactions among individuals [19]. This allows us to generate data on the organizational level from empirical insights gathered on the individual level. For that purpose, we strive for answering the following research question: What is the influence of feedback length and feedback frequency on organizational return of investment (ROI)?

We contribute to theory in several ways. First, we provide descriptive knowledge by shedding light on the aggregation logic of existing individual-level feedback concepts on the organizational level. Second, we are, best to our knowledge, the first studying the interrelationship of feedback length and feedback frequency on an organizational level analysis. Third, we propose that there is a combined sweet spot of rather short and frequent feedbacks, delivered via a feedback app, for maximizing the impact of feedback on the organizational ROI.

We contribute to practice by providing insights for the development of feedback trainings for managers. Furthermore, our study allows developers of feedback apps to derive design features from our findings. For example, an app may help feedback givers in achieving the optimal length for their message or send a reminder when the next feedback is due. These efforts enable organizations to enhance the ROI of their feedback exchange and ultimately build competitive advantage.

\section{Conceptual Foundation}

Our simulation model builds upon three research disciplines. First, feedback as a part of organizational science. Second, the evaluation of the ROI of corporate projects builds upon insights from accounting and finance. Third, research on socio-technical interactions with digital artifacts like feedback apps belong to the realm of information systems research. 


\subsection{Definition of Feedback}

Feedback in the traditional world was conceptualized as information provided by an agent (e.g., manager, colleague, book) regarding aspects of one's performance or understanding. Thus, feedback is a "consequence" of performance [20]. Hence, the purpose of feedback is to assess a state and evaluate its strengths and weaknesses once at the end of the carried-out task [21]. Feedback was not seen as something given along the learning process to incrementally improve performance and support self-reflection over time [22]. Thus, this definition does not explicitly contain the idea that feedback can have multiple purposes, such as motivation, initiation of self-regulated processes or provision of suggestions for improvement in the future. The conceptualization of the purpose of feedback and how it should be provided has changed. Feedback is no longer seen as a one-time event but rather as a process in which employees have an active role to play [23]. Consequently, more recent definitions conceptualize feedback as a process through which employees make sense of information from various sources and use it to enhance their work or learning strategies. Hence, this conception goes beyond notions that feedback is principally about managers or human resources informing employees about strengths, weaknesses and how to improve, but it rather emphasizes the centrality of the employee's role in sense-making and processing the comments to improve subsequent work.

There is a broad body of research around feedback characteristics. For example, scholars distinguish between formal and informal feedback [9]. Furthermore, feedback differs for tasks which require skill or effort [24] and creativity or diligence [25]. Moreover, performance depends on the amount of ambiguity and uncertainty surrounding a particular task [26].

While feedback can be applied in many areas of life, we study it in the context of organizations. Organizations can shape their employees feedback orientation by fostering a feedback culture [8]. Furthermore, organizational feedback develops from a task-based approach to an organizational practice [5]. Therefore, several authors argue that feedback should be studied as a complex product of organizational culture $[8,9$, 27]. One of the reasons organizations provide feedback to their employees to gain competitive advantage [5]. While this shows that feedback can bring positive returns if 
it is applied correctly, it still generates cost. Concretely, providing, reading, and reflecting upon feedback requires time resources from employees which could be used for other productive tasks. However, investments in human capital should be analyzed like any other corporate investment [28]. For this, the measure of ROI can be used as a widely accepted metric throughout business [28]. Phillips [29] proposes a calculation which sets net returns in relation to total investment cost. However, while the value of the investments in human resources can often be determined easily, the benefits are sometimes hard to monetize [30].

\subsection{Characteristics of a Feedback Message in the Context of Feedback Apps}

Feedback can either be provided verbally or in written form. Verbal feedback is mostly delivered face-to-face, which includes body language and intonation [31]. In contrast, written feedback is rather delayed and emotions are often hidden between the lines [32]. To facilitate written feedback, organizations have increasingly adopted feedback apps [33]. Feedback apps are digital work tools, enabling written feedback exchange [2, 33]. Such technological artifacts make it easier for organizations to provide the increased feedback frequency demanded by employees [34].

The length of a feedback is highly correlated with its specificity [34]. Therefore, insights about the relationship between specificity and performance can assumed to be existent for feedback length. While high specificity leads to enhanced performance [35], too lengthy feedbacks might not get read at all [11]. Especially, when feedback is provided frequently, high specificity is not effective [35]. This implies a sweet spot which optimizes specificity and makes sure that the message will be read.

Today's working world is characterized by a dynamically changing environment. Therefore, annual reviews do not fit in anymore [2]. Consequently, large international organizations such as Accenture, Adobe, Goldman Sachs or SAP implement regular check-ins and instant feedback tools [14, 15]. Similarly, scholars suggest that feedback should be provided more often and in an informal way. In particular, the feedback process should follow a continuous nature [36]. Frequent feedback is more effective in improving employee performance than infrequent feedback [35]. However, Holderness, Olsen and Thornock [37] claim that frequent feedback is only able to 
improve performance when employees consent to receiving high-frequency feedback. Hence, feedback frequency has a curvilinear, inverted-U relationship with task performance [38]. But if feedback is provided less frequently, it has to be more detailed to be effective [39]. Furthermore, the frequency base-rate depends on the underlying task that is performed by employees [39].

\section{$3 \quad$ Research Method}

\subsection{Simulation}

The basic idea behind the methodology of computer simulation is mimicking real-word constructs with software code [40]. To achieve this, researchers program connections and interactions between simplified theoretical concepts. This allows them to run experiments with various parameter settings and analyze different outcomes [41]. Agent-based modelling is one such simulation method, which enables quantitative theory development. As the name suggests, it consists of agents, which act upon the given situation by pre-defined behavior rules [19]. This method is particularly useful in conducting 'what-if' analyses by modifying inputs or processes [42]. Consequently, organizational science scholars have accepted the methodology and take advantage of simulation models in their research [43-45].

In developing the agent-based simulation model, this study follows the process proposed by Sargent $[41,45,46]$. First, the theoretical foundation is synthesized from the existing body of literature. These insights are used to build a conceptual model. Based on this conceptual model, the simulation is being implemented. This step includes the calibration and validation of the model. Lastly, experiments with the built model are conducted and the resulting data is being analyzed.

\subsection{Conceptual Model Development}

Next to the theoretical foundations presented in chapter two, the organizational context plays an important role for developing the simulation. Therefore, we collected and analyzed data in a US-bank's call center to build an empirical foundation for the 
simulation model. For this, we introduced a designated feedback app which was built into the agents' workflow. Whenever a ticket was resolved, the manager provided feedback. While it was not mandatory to use the app, the strong integration built a favorable foundation. Our data contains 4'076 feedbacks collected over the period of one year. Feedback exchange happened between 131 unique givers and 181 unique receivers.

This organizational setting makes sense, as the main task of call center agent is to solve tickets. First, solving a ticket can easily be priced by multiplying the required time with the hourly wage of a call center agent. This is often a hurdle in measuring return in organizational settings. Second, this task can be measured and recorded easily. Third, task outcomes are comparable among employees. This allows managers to identify inefficiencies and build feedback recommendations upon these insights. In conclusion, our organizational setting features a task which requires effort and diligence, and managers give informal feedback on it.

Concretely, three simulation model parameters stem from this data. First, to evaluate individual work performance, we use the daily number of solved tickets per call center agent. Second, we have information about the length of feedback messages measured in words. Third, the number of days between feedback interactions gives us the feedback frequency. We analyzed the distribution of these three measures with a kernel density estimation. From this, we derived a function that allows the simulation model to sample data that follow the empirical distribution. By feeding empirical data into the simulation model, our results can be grounded in a more realistic scenario, which safeguards the validity of simulation results.

\subsection{Simulation Development and Validation}

To develop the simulation model, this study utilizes NetLogo [47], a software tool specifically developed for agent-based modelling. This tool has been successfully utilized in previous studies [42] and is able to simulate organizational behavior [48]. 
Agents: The simulation consists of two types of agents. First, managers who are responsible of several subordinates and provide feedback to them. Second, call center agents solving support tickets. In doing so, they receive feedback from their managers.

Interactions: In the beginning of the simulation, all agents are created and configured according to model inputs. Managers are responsible to provide feedback to their assigned employees. This happens after a certain time interval, which is sampled from the empirical model described previously. For this job, they must perform two tasks. First, they need to monitor an employee's work. Second, they need to write the feedback message. Both tasks require a time investment from managers. The monitoring time is randomly drawn, and the writing time is calculated based on the number of words of a feedback and the average duration to write a word. When an employee receives a feedback, the model triggers three actions. First, the employee reads the feedback. Second, she needs to reflect upon the content [18]. Third, she reacts to the feedback [9]. The first two require a time investment by the employee, which follow the same logic as the writing and monitoring of the manager. The reaction is modeled according to the following logic. The first decision is whether the employee accepts the feedback [9]. If she accepts it, she decides whether she is willing to change or not [17]. The former leads to an improved performance in the form of an increased ticket solving speed, the latter implies an unchanged working speed. However, if the employee does not accept the feedback in the first place, she faces another decision. She can either react negatively and reduce her performance or ignore the message and stay at the same output level [17]. Employees' reactions are randomly assigned to them at the beginning of a simulation run. Afterwards, they change it based on assigned probabilities, which reflect different personalities and business events.

After the employee reacted to the feedback, the manager again reacts to the employee's behavior. If the manager recognizes that the employee is changing his or her behavior (both negatively or positively), increases the frequency and length of the feedback message. This implies a higher feedback perceived quality, which in turn leads to improved outcomes [9]. Table 1 summarizes the most important model parameters. 
Table 1. Model Parameters

\begin{tabular}{|c|c|c|c|}
\hline Parameter & Description & Default Value & Justification \\
\hline $\begin{array}{l}\text { Ticket } \\
\text { solving time }\end{array}$ & $\begin{array}{l}\text { The amount of time it takes an } \\
\text { employee to solve a ticket }\end{array}$ & $\begin{array}{l}\text { Dist. from feedback } \\
\text { app }\end{array}$ & $\begin{array}{l}\text { Empirical } \\
\text { data }\end{array}$ \\
\hline $\begin{array}{l}\text { Probability } \\
\text { of behavior } \\
\text { change per } \\
\text { round }\end{array}$ & $\begin{array}{l}\text { Determines the likelihood that } \\
\text { an employee changes his } \\
\text { behavior from the one a } \\
\text { feedback back. }\end{array}$ & $\begin{array}{l}\text { Personality type: } \\
1: 10 \% \\
2: 25 \% \\
3: 50 \%\end{array}$ & $\begin{array}{l}\text { Parameter } \\
\text { testing }\end{array}$ \\
\hline $\begin{array}{l}\text { Feedback } \\
\text { acceptance }\end{array}$ & $\begin{array}{l}\text { Whether or not an employee } \\
\text { accepts a feedback }\end{array}$ & $\begin{array}{l}\text { TRUE or FALSE } \\
\text { according to } \\
\text { behavior change }\end{array}$ & [9] \\
\hline $\begin{array}{l}\text { Willingness } \\
\text { to change }\end{array}$ & $\begin{array}{l}\text { Whether or not an employee is } \\
\text { willing to change }\end{array}$ & $\begin{array}{l}\text { TRUE or FALSE } \\
\text { according to } \\
\text { behavior change }\end{array}$ & [9] \\
\hline $\begin{array}{l}\text { Negative } \\
\text { reaction }\end{array}$ & $\begin{array}{l}\text { Whether or not an employee } \\
\text { shows a negative reaction }\end{array}$ & $\begin{array}{l}\text { TRUE or FALSE } \\
\text { according to } \\
\text { behavior change }\end{array}$ & [49] \\
\hline $\begin{array}{l}\text { Feedback } \\
\text { length }\end{array}$ & $\begin{array}{l}\text { Length of the feedback } \\
\text { message in words }\end{array}$ & $\begin{array}{l}\text { Dist. from feedback } \\
\text { app }\end{array}$ & $\begin{array}{l}\text { Empirical } \\
\text { data }\end{array}$ \\
\hline $\begin{array}{l}\text { Feedback } \\
\text { frequency }\end{array}$ & $\begin{array}{l}\text { The number of days between } \\
\text { consecutive feedback }\end{array}$ & $\begin{array}{l}\text { Dist. from feedback } \\
\text { app }\end{array}$ & $\begin{array}{l}\text { Empirical } \\
\text { data }\end{array}$ \\
\hline $\begin{array}{l}\text { Positive } \\
\text { change }\end{array}$ & $\begin{array}{l}\text { Base rate of improvement } \\
\text { (scaled with length, frequency } \\
\text { and learning effect) }\end{array}$ & 0.5 & $\begin{array}{l}\text { Empirical } \\
\text { data }\end{array}$ \\
\hline $\begin{array}{l}\text { Negative } \\
\text { change }\end{array}$ & $\begin{array}{ll}\text { Performance } & \text { reduction } \\
\text { occurring when } \\
\text { reacts negatively }\end{array}$ & 0.001 & $\begin{array}{l}\text { Parameter } \\
\text { testing }\end{array}$ \\
\hline $\begin{array}{l}\text { Learning } \\
\text { effect }\end{array}$ & $\begin{array}{l}\text { Scales the improvement with a } \\
\text { learning effect }\end{array}$ & $\begin{array}{l}\text { Learning speed } \\
\text { follows a sigmoid- } \\
\text { curve }\end{array}$ & {$[50]$} \\
\hline $\begin{array}{l}\text { Feedback } \\
\text { giver } \\
\text { reaction }\end{array}$ & $\begin{array}{l}\text { How a feedback giver reacts to } \\
\text { recipient's behavior after } \\
\text { receiving feedback }\end{array}$ & $\begin{array}{l}\text { For positive and } \\
\text { negative reactions } \\
\text { increased frequency } \\
\text { and length }\end{array}$ & {$[51]$} \\
\hline $\begin{array}{l}\text { Writing time } \\
\text { per word }\end{array}$ & $\begin{array}{l}\text { How long it takes to write a } \\
\text { word (seconds) }\end{array}$ & Random: 1.5 - 4 & $\begin{array}{l}\text { Based on } \\
\text { average of } \\
\text { adults }\end{array}$ \\
\hline $\begin{array}{l}\text { Monitoring } \\
\text { time }\end{array}$ & $\begin{array}{l}\text { Time to check employee's } \\
\text { work (minutes) }\end{array}$ & Random: 4 - 10 & $\begin{array}{l}\text { Parameter } \\
\text { tests }\end{array}$ \\
\hline $\begin{array}{l}\text { Reading } \\
\text { time per } \\
\text { word }\end{array}$ & $\begin{array}{l}\text { How long it takes to read a } \\
\text { word (seconds) }\end{array}$ & Random: $0.4-1$ & $\begin{array}{l}\text { Based on } \\
\text { average of } \\
\text { adults }\end{array}$ \\
\hline $\begin{array}{l}\text { Reflection } \\
\text { time }\end{array}$ & $\begin{array}{l}\text { Reflect upon the feedback } \\
\text { content (minutes) }\end{array}$ & Random: $2-10$ & $\begin{array}{l}\text { Parameter } \\
\text { tests }\end{array}$ \\
\hline
\end{tabular}


Organizational Setting: We set the number of employees in the simulation in such a way, that they represent a call center team. This allows us to optimize simulation speed while capturing sufficient interactions among workers. Furthermore, the obtained results can be scaled for larger organizations. To control the time dimensions, we set the number of working hours per day (8) and the working days per year (261) to USstandards. As we run the simulation for three years, this translates to 783 ticks.

To account for differences in the value of time for managers and employees, we set an individual hourly wage for each agent type. The validity of the simulation model was analyzed by applying three techniques [46]. First, internal validity tests ensure the consistency of results across different simulation runs with the same setting. The model was calibrated until there was low enough variance in the results across multiple simulation runs. However, some variance is expected, as the various random variables lead to different starting points. Second, degeneracy tests allowed us to set ranges for model parameters. For example, time ranges over more than five years do not produce valid results as the mechanisms of the simulated organization are different in the long run. Similarly, not all employees will ever be willing to change their behavior. Lastly, through sensitivity analysis the effects of the independent variables could be validated. We did this by changing one independent variable at the time ceteris paribus.

\subsection{Simulation Experiments}

All three experiments measure ROI of feedback exchange in the simulated organization. For this, we analyze the simulation results as follows. The measure of return is based on the additional ticket volume the agents solved thanks to the feedback they received. This volume is multiplied with the average ticket solving time. To calculate returns and investments in the same unit, the total time is multiplied by the wage of call center agents. The organization's feedback cost consists of the agent's and manager's time investments as specified in the previous section multiplied with each agent type's wage. This allows us to calculate ROI by subtracting the total costs from the total gains to receive the return and then dividing the result with the total costs. 
Table 2. Simulation Experiments (each simulation run comprised 783 time steps)

\begin{tabular}{ll}
\hline Experiments & Setup \\
\hline Feedback length & We shifted the distribution of the feedback length from 0 to \\
& 800 words in steps of 10 and ran the simulation 50 times per \\
& setting. Thus, the analyses of individual effects were based \\
& on $\mathrm{n}=4,050=81 \times 50$ simulation runs. The feedback \\
& frequency was set to the baseline of the empiric data. \\
& We shifted the distribution of the feedback frequency from \\
& 0 to 125 in steps of 1 and ran the simulation 50 times per \\
Feedback frequency & setting. Thus, the analyses of individual effects were based \\
& on $\mathrm{n}=6,300=126 \times 50$ simulation runs. The feedback \\
& length was set to the baseline of the empiric data. \\
& We shifted the distribution of the feedback length and \\
feedback frequency simultaneously. The length from \\
0 to 800 in steps of 20 and the frequency from 0 to 125 in \\
steps of 5. Then, we ran the simulation 30 times per setting. \\
Thus, the analyses of individual effects were based on $\mathrm{n}=$ \\
$31,980=41 \times 26 \times 30$ simulation runs.
\end{tabular}

This measure represents a ratio that shows how many times a monetary unit invested in feedback exchange is rising financial return from it.

The first experiment varies the independent variable feedback length. To do so, we move the distribution of the kernel density estimation. Therefore, the average sample will be either lower or higher than in the empirical distribution. This allows us to vary the length of feedback messages from managers. Second, we vary the frequency of the feedbacks by again moving the empirical distribution. Finally, we vary both variables simultaneously to study combined effects. Table 2 presents an overview of our experiments. 


\section{Simulation Results}

To analyze the data generated by our simulation experiments, we conducted regression analyses. Hereby, the analysis of our $\mathrm{R}^{2}$-values (Tables 3-5) revealed that non-linear models were significantly better in explaining the relationship between our independent variables and ROI. Therefore, we present the results of our polynomial regression analysis. Due to the highly different magnitude of the independent variables and the dependent variable, coefficients are rather small. While we could normalize independent variables to scale the ratio, we prefer the intuitiveness of the operationalization of feedback length through the number of words and feedback frequency through the amount of days between feedbacks. Furthermore, even small changes in ROI have a significant impact for large organizations.

\subsection{Individual Effects of Feedback Length}

Figure 1 reveals a relationship between feedback length and ROI, which follows an inverted U-shape. Table 3 shows that the length of feedback messages has a significant (all parameters $\mathrm{p}<0.001)$ impact on the ROI of feedback in organizations $\left(\mathrm{R}^{2}=0.215\right)$. Very short feedbacks (0-150 words) provide less return than medium ones (150-450 words). But the longer a feedback message is written, the lower the ROI gets after a tipping point. Hence, the ideal feedback length is medium.

Table 3. Regression Models for the Individual Effects of Feedback Length

\begin{tabular}{llll}
\hline Model & Linear & Quadratic & Cubic \\
\hline Intercept & $1.098^{* * *}$ & $-0.087^{* * *}$ & -0.035 \\
FB-Length & 0.0001 & $0.016^{* * *}$ & $0.024^{* * *}$ \\
FB-Length $^{2}$ & & $-2.279 \mathrm{e}-05^{* * *}$ & $-5.908 \mathrm{e}-05^{* * *}$ \\
FB-Length $^{3}$ & & & $3.456 \mathrm{e}-08^{* * *}$ \\
R $^{2}$ & 0.000 & 0.215 & 0.241 \\
F-Statistic & 4.627 & 4906 & 3807 \\
AIC & $1.830 \mathrm{e}+05$ & $1.744 \mathrm{e}+05$ & $1.731 \mathrm{e}+05$ \\
\hline
\end{tabular}

Notes: $* \mathrm{p}<0.01 ; * * \mathrm{p}<0.005 ; * * * \mathrm{p}<0.001$ 

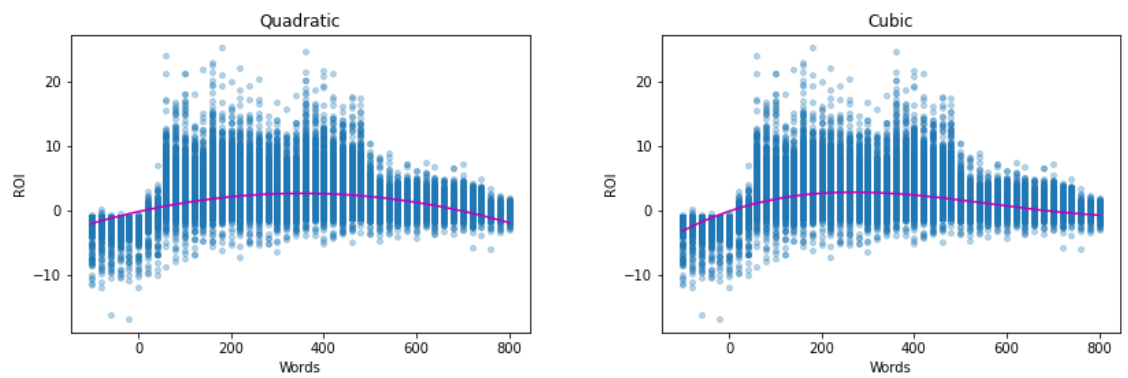

Figure 1. Individual Effects of Feedback Length

\subsection{Individual Effects of Feedback Frequency}

While the cubic model provides a better fit for the feedback length, figure 2 reveals that for feedback frequency, the quadratic and cubic model are very similar. Both show a falling ROI for larger delays between feedbacks. Therefore, table 4 indicates that ROI is highest when organizations provide frequent feedback $\left(\mathrm{R}^{2}=0.206\right)$.

Table 4. Regression Models for the Individual Effects of Feedback Frequency

\begin{tabular}{llll}
\hline Model & Linear & Quadratic & Cubic \\
\hline Intercept & $1.313 * * *$ & $2.124 * * *$ & $2.253 * * *$ \\
FB-Frequency & $-0.023 * * *$ & $-0.063 * * *$ & $-0.075 * * *$ \\
FB-Frequency $^{2}$ & & $0.0003 * * *$ & $0.0006 * * *$ \\
FB-Frequency $^{3}$ & & & $-1.361 \mathrm{e}-06$ \\
$\mathrm{R}^{2}$ & 0.173 & 0.206 & 0.207 \\
F-Statistic & 1320 & 819.2 & 548.2 \\
AIC & $2.565 \mathrm{e}+04$ & $2.540 \mathrm{e}+04$ & $2.539 \mathrm{e}+04$ \\
\hline
\end{tabular}

Notes: ${ }^{*} \mathrm{p}<0.01 ; * * \mathrm{p}<0.005 ; * * * \mathrm{p}<0.001$ 

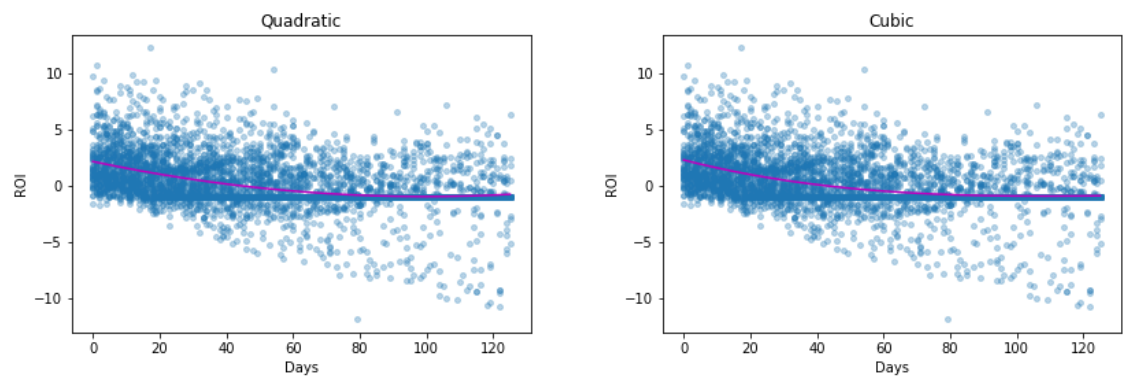

Figure 2. Individual Effects of Feedback Frequency

Table 5. Regression Models for the Joint Effects of Feedback Length \& Frequency

\begin{tabular}{|c|c|c|c|}
\hline Model & Linear & Quadratic & Cubic \\
\hline Intercept & $5.068 * * *$ & $5.938 * * *$ & $4.942 * * *$ \\
\hline FB-Length & $-0.004 * * *$ & $0.003 * * *$ & $0.024 * * *$ \\
\hline FB-Length ${ }^{2}$ & & $-1.333 \mathrm{e}-05^{* * *}$ & $-7.494 \mathrm{e}-05^{* * *}$ \\
\hline FB-Length ${ }^{3}$ & & & $4.631 \mathrm{e}-08 * * *$ \\
\hline FB-Frequency & $-0.032 * * *$ & $-0.100 * * *$ & $-0.133 * * *$ \\
\hline FB-Frequency ${ }^{2}$ & & $0.0004 * * *$ & $0.0009 * * *$ \\
\hline FB-Frequency ${ }^{3}$ & & & $-1.342 \mathrm{e}-06^{* * *}$ \\
\hline Length $\times$ Frequency & & $5.245 \mathrm{e}-05 * * *$ & $5.189 \mathrm{e}-05^{* * *}$ \\
\hline Length $^{2} \times$ Frequency & & & $9.643 \mathrm{e}-08 * * *$ \\
\hline Length $\times$ Frequency $^{2}$ & & & $-6.127 \mathrm{e}-07 * * *$ \\
\hline $\mathrm{R}^{2}$ & 0.249 & 0.343 & 0.375 \\
\hline F-Statistic & 5299 & 3345 & 2135 \\
\hline AIC & $1.532 \mathrm{e}+05$ & $1.489 \mathrm{e}+05$ & $1.473 \mathrm{e}+05$ \\
\hline
\end{tabular}

Notes: $* \mathrm{p}<0.01 ; * * \mathrm{p}<0.005 ; * * * \mathrm{p}<0.001$

\subsection{Joint Effects of Feedback Length and Feedback Frequency}

The quadratic model (table 5) suggests that frequent (0-21 days), medium-length feedback boasts the highest ROI potential for organizations $\left(\mathrm{R}^{2}=0.343\right)$. The effect of feedback length is much stronger in shorter frequencies than for longer time-periods 
between feedbacks. The cubic model reveals another level of complexity. While the sweet spot is also for medium-length, frequent feedback, there is another high-point for infrequent feedback which is long (figure 3). Furthermore, the frequency shows a higher sensitivity than in the quadratic model. The low point is represented by long feedbacks that are sent very frequently.
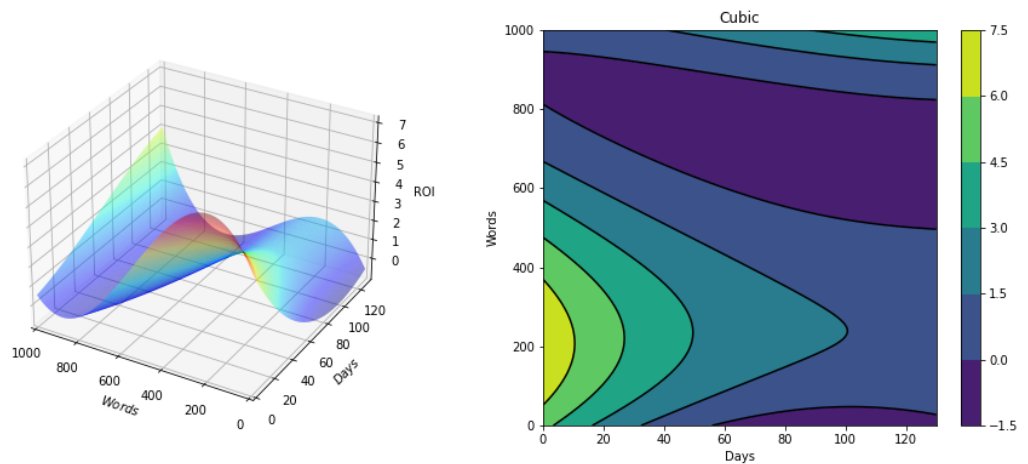

Figure 3. Joint Effects of Feedback Length \& Frequency

\section{Discussion}

In the past feedback was seen as an annual management process in which managers provide feedback to their employees for the entire year. However, the world isn't really on an annual cycle anymore for anything [16]. Employees and the generation $Y$ request to receive more and timely feedback from their managers. Nevertheless, the trend of more and more feedback has hardly been challenged in the literature, since measuring this effect on an organizational level is highly complex and problematic. Hence, the question arises as how much feedback is necessary and beneficial for organizations. Therefore, we explored the organizational effects of feedback through well-grounded computer simulation experiments in a specific task setting. This limits the generalizability of our results to a subset of tasks and organizations.

Our study presents several findings. First, we show that the feedback length has a curve-linear relationship with ROI of feedback exchange that follows an inverse Ushape. This implies that there is a sweet spot for feedback length when optimizing ROI. 
While very short messages do not suffice in delivering enough specificity, too long feedbacks might not get read or overwhelm recipients. This is consistent with previous literature [39]. However, our findings extend the current knowledge as they measure the impact not only on performance but on ROI. This is important because the performance gain must be financially justified [28].

Second, feedback frequency has a negative relationship with ROI. The less frequent employees get feedback, the lower is the return for the organization. While the cost is low, the performance is not high either. This implies that if feedback cannot be provided frequently, the resources might not be worthwhile, and the investment should be rejected. Nevertheless, some of our findings are not consistent with literature. While Casas-Arce et al. [39] describe an inverted U-shape relationship between frequency and performance, we present a falling relationship. However, we analyze another dependent variable which is conceptualized as ROI. Furthermore, Casas-Arce et al. [39] acknowledged that the relationship may alter for different tasks.

Third, we analyzed the joint effects of feedback length and feedback frequency. Our analysis shows that when the days between consecutive feedbacks are low, the length of the feedback has a large effect. If the frequency is smaller, the impact of a change in length is much lower. Moreover, Figure 3 shows two optimal points. One represents frequent, medium-length feedback. The other less frequent, but long feedback. This might seem contradictory as we have previously shown a negative relationship between ROI and feedback frequency when analyzed isolated. However, this second optimum can be understood as very resource effective. Because feedback is not given often, the associated costs are low. Hence, smaller improvements still have a positive ROI.

This study's findings have several theoretical and practical implications. First, it extends existing literature by studying individual level effects of feedback exchange on the organizational level. This allows us to challenge the assumption that more feedback is always better. Our study shows that organizations must analyze their investments in feedback apps to gain the expected benefits. Second, while past research revealed interactions of feedback length and feedback frequency on the individual level, our study is, best to our knowledge, the first to shed a light on joint effects of these two feedback characteristics. Third, we revealed that for maximizing ROI, organizations 
must motivate their employees to write rather short feedback and provide it frequently. While it requires more time resources than annual feedbacks, the return makes the investment worthwhile.

We contribute to practice in three ways. g Second, app developers can derive design choices from our insights. For example, a feedback app can highlight whether a feedback message contains enough words while it is written. Additionally, managers could receive push messages when the next feedback for an employee is due. Third, managers must closely analyze organizational feedback exchange and adjust their strategy by analyzing the ROI of their efforts. Our insights provide them guidance in doing so.

\section{Conclusion and Limitations}

No study comes without limitations. First, to operationalize the ROI we selected ticket solving speed as measure of return. While this allows us to overcome the hurdle of monetizing a cultural investment [30], we ignore other important factors. For example, the quality of the solved ticket plays an equally important role for long-term success. Further studies could analyze the ROI with a focus on quality. Second, as any model we had to abstract from the conceptual foundations. For instance, our simulation model assumes that all employees stay with the organization. This is not true in practice and might have an impact on ROI as organizations invest in resources they will not possess in the future and therefore, cannot profit from arising competitive advantages. Third, our results are only valid for a certain type of task. Solving tickets is a relatively easy and repetitive task. In contrast, tasks such as drug discovery, creative work or legal counselling are more complex and do not follow the same logic. Therefore, further studies need to analyze the impact of task type on the ROI of feedback.

In conclusion, our findings have significant implications for both theory and practice. We show that organizations can optimize ROI from feedback exchange by varying the feedback length and frequency. While feedback length shows an inverted U-shape relationship with ROI, feedback frequency is negatively correlated. When analyzed jointly, medium-length, frequent feedback and infrequent, longer feedback represent ROI sweet spots. 


\section{References}

1. Barth, L.: Wie Unternehmen eine attraktive, aber anspruchsvolle neue Generation von Potenzialträgern begeistern und binden können, https://www.egonzehnder.com/de/insight/werben-um-die-generation-y

2. Levy, P.E., Tseng, S.T., Rosen, C.C., Lueke, S.B.: Performance Management: A Marriage between Practice and Science - Just Say "I do". In: Buckley, M.R., Wheeler, R.A., Halbesleben, R.B.J. (eds.) Research in Personnel and Human Resources Management, pp. 155-213. Emerald Publishing Limited (2017)

3. Lechler, R., Stoeckli, E., Rietsche, R., Uebernickel, F.: Looking Beneath the Tip of the Iceberg: The Two-Sided Nature of Chatbots and Their Roles for Digital Feedback Exchange. In: Proceedings of the 27th European Conference on Information Systems (ECIS 2019) (ed.) (2019)

4. Carmeli, A., Weisberg, J.: Exploring turnover intentions among three professional groups of employees. Human Resource Development International 9, 191-206 (2006)

5. Baker, N.: Employee feedback technologies in the human performance system. Human Resource Development International 13, 477-485 (2010)

6. Ilgen, D.R., Fisher, C.D., Taylor, M.S.: Consequences of individual feedback on behavior in organizations. Journal of Applied Psychology 64, 349-371 (1979)

7. Ilgen, D.R., Barnes-Farrell, J.L., McKellin, D.B.: Performance Appraisal Process Research in the 1980s: What Has It Contributed to Appraisals in Use? Organizational Behavior and Human Decision Processes 54, 321-368 (1993)

8. London, M., Smither, J.W.: Feedback orientation, feedback culture, and the longitudinal performance management process. Human Resource Management Review 12, 81-100 (2002)

9. H. Mulder, R., D. Ellinger, A.: Perceptions of quality of feedback in organizations. European Journal of Training and Development 37, 4-23 (2013)

10. Narciss, S.: Feedback Strategies for Interactive Learning Tasks. In: Spector, J.M., Merrill, M.D., Elen, J., Bishop, M.J. (eds.) Handbook of Research on Educational Communications and Technology, pp. 125-144. Springer, New York (2008)

11. Shute, V.J.: Focus on Formative Feedback. Review of Educational Research 78, 153-189 (2008)

12. Tata, J.: The Influence of Managerial Accounts on Employees' Reactions to Negative Feedback. Group \& Organization Management 27, 480-503 (2002)

13. Buckingham, M., Goodall, A.: Reinventing Performance Management. Harvard Business Review 93, 40-50 (2015)

14. Cappelli, P., Tavis, A.: The Performance Management Revolution. Harvard Business Review 94, 58-67 (2016)

15. Pulakos, E.D., Hanson, R.M., Arad, S., Moye, N.: Performance Management Can Be Fixed: An On-the-Job Experiential Learning Approach for Complex Behavior Change. Industrial and Organizational Psychology 8, 51-76 (2015)

16. Nisen, M.: The management cliche you really can't afford to ignore. Quartz (2015)

17. Raemdonck, I., Strijbos, J.-W.: Feedback perceptions and attribution by secretarial employees. Euro J of Training and Dev 37, 24-48 (2013)

18. Anseel, F., Lievens, F., Schollaert, E.: Reflection as a strategy to enhance task performance after feedback. Organizational Behavior and Human Decision Processes 110, 23-35 (2009) 
19. Bonabeau, E.: Agent-based modeling: Methods and techniques for simulating human systems. Proceedings of the National Academy of Sciences 99, 7280-7287 (2002)

20. Hattie, J., Timperley, H.: The Power of Feedback. Review of Educational Research 77, 81-112 (2007)

21. Schleicher, D.J., Baumann, H.M., Sullivan, D.W., Levy, P.E., Hargrove, D.C., BarrosRivera, B.A.: Putting the System Into Performance Management Systems: A Review and Agenda for Performance Management Research. Journal of Management 44, 2209-2245 (2018)

22. Ashford, S.J., Tsui, A.S.: Self-Regulation For Managerial Effectiveness: The Role Of Active Feedback Seeking. Academy of Management Journal 34, 251-280 (1991)

23. Dawson, P., Henderson, M., Mahoney, P., Phillips, M., Ryan, T., Boud, D., Molloy, E.: What makes for effective feedback: staff and student perspectives. Assessment \& Evaluation in Higher Education 44, 25-36 (2019)

24. Baumeister, R.F., Hutton, D.G., Cairns, K.J.: Negative Effects of Praise on Skilled Performance. Basic and Applied Social Psychology 11, 131-148 (1990)

25. Stobbeleir, K.E.M. de, Ashford, S.J., Buyens, D.: Self-Regulation of Creativity at Work: The Role of Feedback-Seeking Behavior in Creative Performance. AMJ 54, 811-831 (2011)

26. London, M., Mone, E.M.: Performance management: Processes that reflect and shape organizational culture and climate. The Oxford Handbook of Organizational Climate and Culture, 79 (2014)

27. Dahling, J.J., O’Malley, A.L.: Supportive Feedback Environments Can Mend Broken Performance Management Systems. Ind. Organ. Psychol. 4, 201-203 (2011)

28. Avolio, B.J., Avey, J.B., Quisenberry, D.: Estimating return on leadership development investment. The Leadership Quarterly 21, 633-644 (2010)

29. Phillips, J.J.: Return on investment in training and performance improvement programs. Butterworth-Heinemann, Amsterdam, Boston (2003)

30. Murray, L.W., Efendioglu, A.M.: Valuing the investment in organizational training. Ind and Commercial Training 39, 372-379 (2007)

31. Kulik, J.A., Kulik, C.-L.C.: Timing of Feedback and Verbal Learning. Review of Educational Research 58, 79 (1988)

32. Kulhavy, R.W.: Feedback in Written Instruction. Review of Educational Research 47, 211 (1977)

33. Stöckli, E., Uebernickel, F., Brenner, W., Weierich, A., Hess, S.: Digital Feedback for Digital Work? Affordances and Constraints of a Feedback App at InsurCorp. In: Proceedings of the International Conference on Wirtschaftsinformatik (WI) (ed.) (2019)

34. Miller, J.S.: High tech and high performance: Managing appraisal in the information age. Journal of Labor Research 24, 409-424 (2003)

35. Park, J.-A., Johnson, D.A., Moon, K., Lee, J.: The Interaction Effects of Frequency and Specificity of Feedback on Work Performance. Journal of Organizational Behavior Management 39, 164-178 (2019)

36. Pulakos, E.D., O’Leary, R.S.: Why Is Performance Management Broken? Ind. Organ. Psychol. 4, 146-164 (2011)

37. Holderness, D.K., Olsen, K.J., Thornock, T.A.: Assigned versus Chosen Relative Performance Information: The Effect of Feedback Frequency on Performance. Journal of Management Accounting Research 32, 137-158 (2020)

38. Lam, C.F., DeRue, D.S., Karam, E.P., Hollenbeck, J.R.: The impact of feedback frequency on learning and task performance: Challenging the "more is better" assumption. Organizational Behavior and Human Decision Processes 116, 217-228 (2011) 
39. Casas-Arce, P., Lourenço, S.M., Martínez-Jerez, F.A.: The Performance Effect of Feedback Frequency and Detail: Evidence from a Field Experiment in Customer Satisfaction. Journal of Accounting Research 55, 1051-1088 (2017)

40. Law, A.M., Kelton, W.D.: Simulation Modeling and Analysis. McGraw-Hill, New York (1991)

41. Davis, J.P., Eisenhardt, K.M., Bingham, C.B.: Developing Theory Through Simulation Methods. AMR 32, 480-499 (2007)

42. Nan, N., Tanriverdi, H.: Unifying the Role of IT in Hyperturbulence and Competitive Advantage Via a Multilevel Perspective of IS Strategy. MIS Quarterly 41, 937-958 (2017)

43. Burton, R.M., Obel, B.: Computational Modeling for What-Is, What-Might-Be, and WhatShould-Be Studies-And Triangulation. Organization Science 22, 1195-1202 (2011)

44. Fioretti, G.: Agent-Based Simulation Models in Organization Science. Organizational Research Methods 16, 227-242 (2013)

45. Haki, K., Beese, J., Aier, S., Winter, R.: The Evolution of Information Systems Architecture: An Agent-Based Simulation Model. MIS Quarterly 44, 155-184 (2020)

46. Kuhl, M.E., Steiger, N.M., Armstrong, F.B., Jones, J.A. (eds.): Proceedings of the 2005 Winter Simulation Conference. IEEE, Piscataway, NJ (2005)

47. Wilensky, U.: NetLogo. Center for Connected Learning and Computer-Based Modeling, Northwestern University, Evanston, IL (1999)

48. Abar, S., Theodoropoulos, G., Lemarinier, P., O'Hare, G.: Agent Based Modelling and Simulation tools: A review of the state-of-art software. Computer Science Review, 13-33 (2017)

49. Murphy, K.R.: Performance evaluation will not die, but it should. Human Resource Management Journal 30, 13-31 (2020)

50. Leibowitz, N., Baum, B., Enden, G., Karniel, A.: The exponential learning equation as a function of successful trials results in sigmoid performance. Journal of Mathematical Psychology 54, 338-340 (2010)

51. Elicker, J.D., Levy, P.E., Hall, R.J.: The Role of Leader-Member Exchange in the Performance Appraisal Process. Journal of Management 32, 531-551 (2006) 